\title{
Author Correction: Rapid multi-directed cholinergic transmission in the central nervous system
}

\author{
Santhosh Sethuramanujam, Akihiro Matsumoto (1), Geoff deRosenroll, Benjamin Murphy-Baum, \\ Claudio Grosman, J. Michael McIntosh, Miao Jing, Yulong Li (iD, David Berson, Keisuke Yonehara (1) \& \\ Gautam B. Awatramani (1)
}

Correction to: Nature Communications https://doi.org/10.1038/s41467-021-21680-9, published online 2 March 2021.

The original version of this Article omitted from the author list the 5th author Claudio Grosman, who is from the Department of Molecular and Integrative Physiology, 407 S. Goodwin Ave, Urbana, IL 61801, USA. Additionally, the Author contributions (G.D. and C.G. constructed and tested kinetic nAChR models.) and Acknowledgements (C.G. (R01-NS042169)) were updated to include C.G.'s contribution. This has been corrected in both the PDF and HTML versions of the Article.

Author contributions: S.S. conducted all the electrophysiological and starburst imaging experiments shown in Figs. 2-6. A.M. performed the ACh imaging experiments shown in Fig. 7. Y.L. and M.J. developed ACh sensor; J.M.M. developed the nAChR antagonist; D.B. conducted all the SBEB analysis in Fig. 1; K.Y. designed AAVs constructs and ACh imaging experiments; G.D. and C.G. constructed and tested kinetic nAChR models; B.M.B. wrote new software for image analysis. G.B.A. and S.S. conceived the experiments and wrote the original manuscript.

Santhosh Sethuramanujam ${ }^{1}$, Akihiro Matsumoto ${ }^{2}$, Geoff deRosenroll ${ }^{1}$, Benjamin Murphy-Baum ${ }^{1}$, Claudio Grosman ${ }^{3}$, J. Michael McIntosh $^{4,5,6}$, Miao Jing ${ }^{7}$, Yulong Li ${ }^{7}$, David Berson ${ }^{8}$, Keisuke Yonehara ${ }^{2 *}$, and Gautam B. Awatramani ${ }^{*}$

${ }^{1}$ Department of Biology, University of Victoria, Victoria, BC V8W 3N5, Canada

2 Danish Research Institute of Translational Neuroscience - DANDRITE, Nordic-EMBL Partnership for Molecular Medicine, Department of Biomedicine, Aarhus University, Ole Worms Allé 8, 8000 Aarhus C, Denmark

${ }^{3}$ Department of Molecular and Integrative Physiology, 407 S. Goodwin Ave, Urbana, IL 61801, USA

${ }^{4}$ George E. Whalen Veterans Affairs Medical Center, ${ }^{5}$ Department of Psychiatry; ${ }^{6}$ School of Biological Sciences, University of Utah, Salt Lake City, UT, USA

7 State Key Laboratory of Membrane Biology, Peking University School of Life Sciences, Beijing 100871, China

${ }^{8}$ Neuroscience, Brown University, 185 Meeting Street, Providence, RI 02912, USA

\section{Acknowledgements}

We thank Dr. Kevin Briggman for making previous SBEM datasets available for further analysis; Dr. Kerry Delaney for his critical feedback on the manuscript; Tracey Michaels for performing AAV injections and help with mouse colony management; Zoltan Raics for developing our visual stimulation system; Bjarke Thomsen and Misugi Yonehara for their technical assistance; Dr. Marla Feller for nGFP mice; and Dr. Jamie Boyd for his help with IGOR software for 2P imaging. This work was supported by grants awarded to A.M.

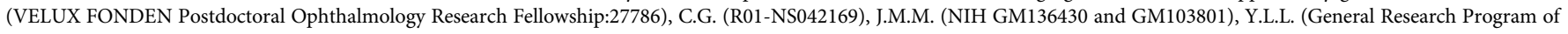
National Natural Science Foundation of China (project 31671118), the NIH BRAIN Initiative (grant U01NS103558), the Beijing Brian Initiative of Beijing Municipal Science \& Technology Commission (Z181100001518004), the Junior Thousand Talent Program of China, and by grants from the Peking-Tsinghua Center for Life Sciences and the State Key Laboratory of Membrane Biology at Peking University School of Life Science), D.B. (RO1 EY012793-19), K.Y. (Lundbeck Foundation: DANDRITE-R248-2016-2518; R252-2017-1060, Novo Nordisk Foundation, NNF15OC0017252, Carlsberg Foundation, CF17-0085, and European Research Council Starting, 638730), and G.B.A. (CIHR 159444). 
(c) Open Access This article is licensed under a Creative Commons Attribution 4.0 International License, which permits use, sharing, adaptation, distribution and reproduction in any medium or format, as long as you give appropriate credit to the original author(s) and the source, provide a link to the Creative Commons license, and indicate if changes were made. The images or other third party material in this article are included in the article's Creative Commons license, unless indicated otherwise in a credit line to the material. If material is not included in the article's Creative Commons license and your intended use is not permitted by statutory regulation or exceeds the permitted use, you will need to obtain permission directly from the copyright holder. To view a copy of this license, visit http://creativecommons.org/licenses/by/4.0/.

(C) The Author(s) 2021 